\title{
HyU: Hybrid Unmixing for longitudinal in vivo imaging of low signal to noise fluorescence
}

Hsiao Chiang

University of Southern California

Daniel Koo

University of Southern California

Masahiro Kitano

University of Southern California

Jay Unruh

Stowers Institute for Medical Research

Le Trinh

University of Southern California

Scott Fraser

University of Southern California

Francesco Cutrale ( $\square$ francesco.cutrale@gmail.com )

University of Southern California https://orcid.org/0000-0003-0517-3069

\section{Methods Article}

Keywords:

Posted Date: January 12th, 2022

DOI: https://doi.org/10.21203/rs.3.rs-1073331/v1

License: (c) (i) This work is licensed under a Creative Commons Attribution 4.0 International License.

Read Full License 


\title{
Title: HyU: Hybrid Unmixing for longitudinal in vivo imaging of low signal to noise fluorescence
}

Authors: Hsiao Ju Chiang ${ }^{1,2, \dagger}$, Daniel E.S. Koo ${ }^{1,2, \dagger}$, Masahiro Kitano ${ }^{1,3}$, Jay Unruh ${ }^{4}$, Le A. Trinh $^{1,2,3}$, Scott E. Fraser ${ }^{1,2,3}$, Francesco Cutrale ${ }^{1,2,3^{*}}$

\author{
Affiliations: \\ ${ }^{1}$ Translational Imaging Center, University of Southern California, Los Angeles, CA \\ ${ }^{2}$ Department of Biomedical Engineering, University of Southern California, Los Angeles, CA \\ ${ }^{3}$ Molecular and Computational Biology, University of Southern California, Los Angeles, CA \\ ${ }^{4}$ Stowers Institute for Medical Research, Kansas City, MO
}

10

*Correspondence to: cutrale@usc.edu

$\dagger$ Equal contribution

\begin{abstract}
The expanded application of fluorescence imaging in biomedical and biological research towards more complex systems and geometries requires tools that can analyze a multitude of components at widely varying time- and length-scales. The major challenge in such complex imaging experiments is to cleanly separate multiple fluorescent labels with overlapping spectra from one another and background autofluorescence, without perturbing the sample with high levels of light. Thus, there is a requirement for efficient and robust analysis tools capable of quantitatively separating these signals.

In response, we have combined multispectral fluorescence microscopy with hyperspectral phasors and linear unmixing to create Hybrid Unmixing (HyU). Here we demonstrate its capabilities in the dynamic imaging of multiple fluorescent labels in live, developing zebrafish embryos. HyU is more sensitive to low light levels of fluorescence compared to conventional linear unmixing approaches, permitting better multiplexed volumetric imaging over time, with less bleaching. HyU can also simultaneously image both bright exogenous and dim endogenous labels because of its high dynamic range. This allows studies of cellular behaviors, tagged components, and cell metabolism within the same specimen, offering a powerful window into 30 the orchestrated complexity of biological systems.
\end{abstract}

One Sentence Summary: Hybrid Unmixing offers enhanced imaging of multiplexed fluorescence labels, enabling longitudinal imaging of multiple fluorescent signals with reduced illumination intensities. 


\section{Main Text:}

\section{Introduction}

In recent years, high-content imaging approaches have been refined for decoding the complex and dynamical orchestration of biological processes. ${ }^{1,2,3}$ Fluorescence, with its high contrast, high specificity and multiple parameters, has become the reference technique for imaging. ${ }^{4,5}$ Continuous improvements in fluorescent microscopes ${ }^{6-9}$ and the ever-expanding palette of genetically-encoded and synthesized fluorophores have enabled the labeling and observation of a large number of molecular species ${ }^{10,11}$. This offers the potential of using multiplexed imaging to follow multiple labels simultaneously in the same specimen, but the technologies for this have fallen short of their fully imagined capabilities. Standard fluorescence microscopes collect multiple images sequentially, employing different excitation and detection bandpass filters for each label. Recently developed techniques allow for massive multiplexing by utilizing sequential labeling of fixed samples but are not suitable for in vivo imaging. ${ }^{12,13}$ Unfortunately, these approaches are ill-suited to separating overlapping fluorescence emission signals, and the narrow bandpass optical filters used to increase selectivity, decrease the photon efficiency of the imaging. (Figs. S1, S2) These limitations have restricted the number of imaged fluorophores per sample (usually 3-4) and risks exposing the specimen to damaging levels of exciting light. This has been a significant obstacle for the dynamic imaging, and has prevented in vivo imaging from reaching its full potential.

Hyperspectral Fluorescent Imaging (HFI) potentially overcomes the limitations of overlapping emissions by expanding signal detection into the spectral domain. ${ }^{14}$ HFI captures a spectral profile from each pixel, resulting in a hyperspectral cube (x,y, wavelength) of data, that can be processed to deduce the labels present in that pixel. Linear unmixing (LU) has been widely utilized to analyze HFI data, and has performed well with bright samples emitting strong signals from fully-characterized, extrinsic fluorophores such as fluorescent proteins and dyes ${ }^{15-17}$. However, in vivo fluorescence microscopy is almost always limited in the number of photons collected per pixel (due to the expression levels, the bio-physical fluorescent properties, and the sensitivity of the detection system), which reduces the quality of the spectra acquired.

A further challenge which affects quality of spectra is the presence of multiple forms of noise in the imaging of the sample. Two examples of instrumental noise are photon noise and read noise. Photon noise, also known as Poisson noise, is an inherent property related to the statistical variation of photons emission from a source and of detection. Poisson noise is inevitable when imaging fluorescent dyes and is more pronounced in the low-photon regime. It poses challenges especially in live and time lapse imaging, where the power of the exciting laser is reduced to avoid photo-damage to the sample, decreasing the amount of fluorescent signal. Read noise arises from voltage fluctuations in microscopes operating in analog mode, during the conversion from photon to digital levels intensity and commonly affects fluorescence imaging acquisition. Most biological samples used for in vivo microscopy are labelled using extrinsic signals from fluorescent proteins or probes but often include intrinsic signals (autofluorescence).

Autofluorescence contributes photons that are undesired, difficult to identify and to account for in LU. The cumulative presence of noise inevitably leads to a degradation of acquired spectra during imaging. As a result, the spectral separation by LU is often compromised, and the Signal to Noise ratio (SNR) of the final unmixing is often reduced by the weakest of the signals 
detected. ${ }^{16}$ Increasing the amount of laser excitation can partially overcome these challenges, but the higher energy deposition in the sample causes photo-bleaching and -damage, affecting both the integrity of the live sample and the duration of the observation. Traditional unmixing strategies such as LU are computationally demanding, requiring long analyses and often slowing the experiment. Combined, these compromises have reduced both the overall multiplexing capability and the adoption of HFI multiplexing technologies.

We have developed Hybrid Unmixing (HyU) as an answer to the challenges that have limited the wider acceptance of HFI for in vivo imaging. HyU employs the phasor approach ${ }^{18}$ merged with traditional unmixing algorithms to more rapidly and more accurately untangle the fluorescent signals from multiple exogenous and endogenous labels. The phasor approach ${ }^{18}$, a popular dimensionality reduction approach for the analysis of both fluorescence lifetime and spectral image analysis ${ }^{19-21}$ provides key advantages to HyU, including spectral compression, denoising, and computational reduction. HyU pairs phasor processing with unmixing algorithms, such as LU, to provide unsupervised analysis of HFI data, removing user subjectivity. Our results show that HyU offers three key advantages: (1) improved unmixing over conventional LU, especially for low intensity images, down to 5 photons per spectra; (2) simplified identification of independent spectral components; (3) dramatically faster processing of large datasets, overcoming the typical unmixing bottleneck for in vivo fluorescence microscopy.

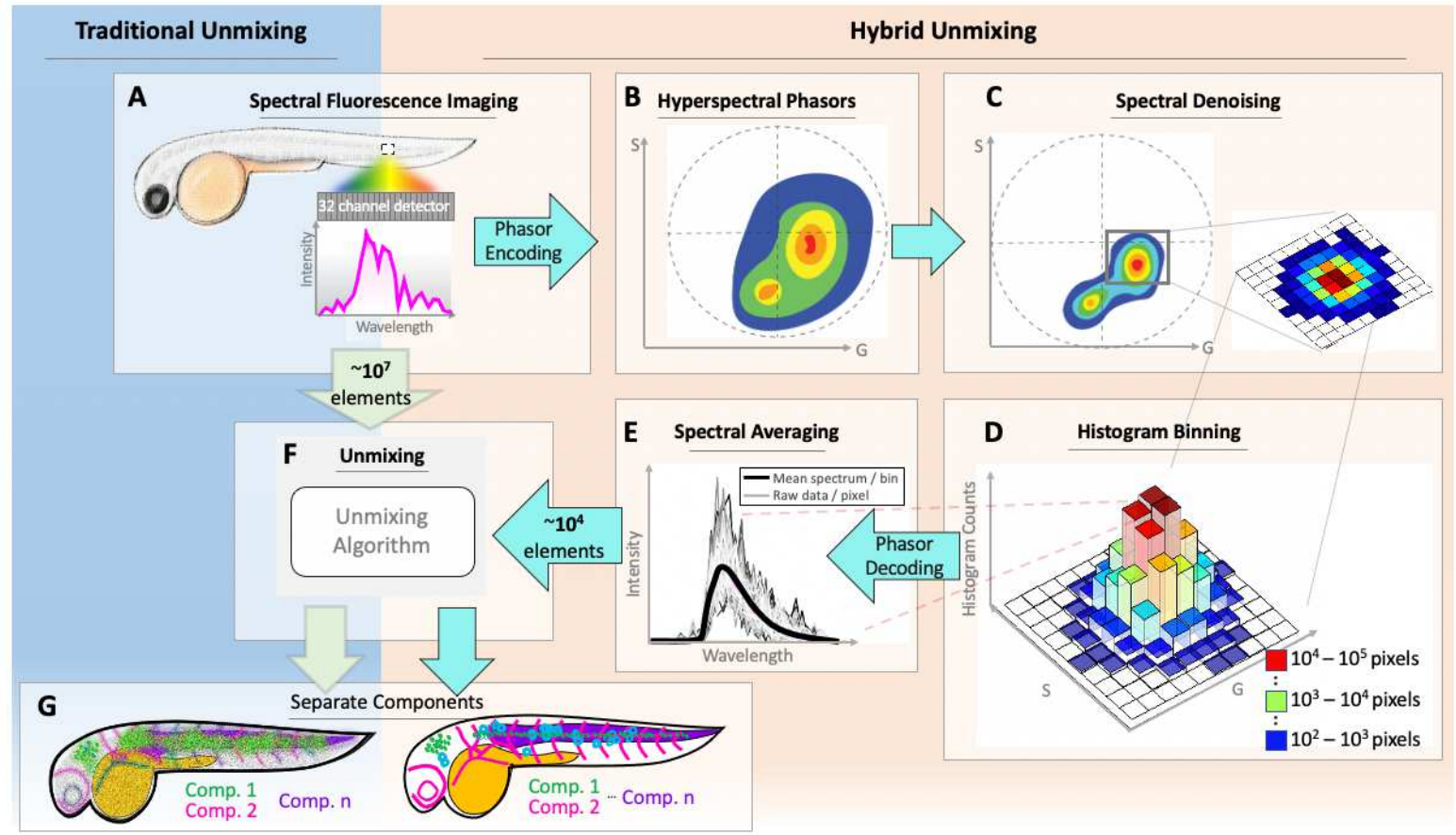

Figure 1. Schematic illustrating how Hybrid Unmixing (HyU) enhances analysis of multiplexed hyperspectral fluorescent signals in vivo. (A) Multicolor fluorescent biological sample (here a zebrafish embryo) is imaged in hyperspectral mode, collecting the fluorescence spectrum of each voxel in the specimen. (B) HyU represents spectral data as a phasor plot, a 2D histogram of the real and imaginary Fourier components (at a single harmonic). (C) Spectral denoising filters reduce the Poisson and instrumental noise on the phasor histogram, providing the first signal improvement. (D) The phasor acts as an encoder, where each histogram-bin corresponds to a number $\mathrm{n}$ of 
pixels, each with a relatively similar spectrum (E). Summing these spectra effectively averages the spectra for that phasor position.

This denoising results in cleaner average spectrum for this set of pixels, which are ideally suited for analytical decomposition through unmixing algorithms (F). (G) Unmixing results in images that separated into spectral components. Here, linear unmixing (LU) is used for unmixing, but $\mathrm{HyU}$ is compatible with any unmixing algorithm.

Note that HyU offers a major reduction in data size and complexity of the LU (or any other unmixing) computation, because the calculation is applied to the $10^{4}$ histogram bins (D), rather the the $\sim 10^{7}$ voxels in the specimen (A). This reduces the number of calculations required for LU dramatically.

\section{Results}

HyU combines the best features of hyperspectral phasor analysis and linear unmixing (LU), resulting in faster computation speeds and more reliable results, especially at low light levels. Phasor approaches reduce the computational load because they are compressive, reducing the 32 channels of an HFI spectral plot into a position on a 2D-histogram, representing the real and imaginary Fourier components of the spectrum (Fig. 1A,B). Different 32 channel spectra are represented as different positions on the $2 \mathrm{D}$ phasor plot, and mixtures of the two spectra will be rendered at a position along a line connecting the pure spectra. Because the spectral content of an entire 2D or 3D image set is rendered on a single phasor plot, there is a dramatic data compression - from a spectrum for each voxel in an image set (up to or even beyond Gigavoxels) to a histogram value on the phasor plot (Megapixels). In addition, because each "bin" on the phasor plot histogram corresponds to multiple voxels with highly similar spectral profiles, the binning itself represents spectral averaging, which reduces the Poisson and instrumental noise (Fig. 1C-E). Poisson noise in the collected light is unavoidable in HFI unless the excitation is turned so high that the statistics of collected fluorescence creates hundreds or thousands of photons per spectral bin. The clear separation of the spectral phasor plot and its referenced imaging data, permits denoising algorithms to be applied to phasor plot with minimal degradation of the image resolution. LU or other unmixing approaches can be applied to the spectra on the phasor plot, offering a dramatic reduction in computational burden for large image data sets (Fig. 1D). To understand this saving, consider the conventional approach of LU applied to image data at the voxel level (Fig. 1A,F). A timelapse volumetric dataset of 512x768x17 (x, $\mathrm{y}, \mathrm{z}$ ) pixels, over 6 timepoints, (Sup. table 1), would require 40 million operations. HyU's requires only $\sim 18$ thousand operations to unmix each bin on the phasor plot, representing more than a thousand-fold saving (Fig. 1F,G).

To quantitatively assess the relative performance of $\mathrm{LU}$ and $\mathrm{HyU}$, we analyzed them on synthetic hyperspectral fluorescent datasets, created by computationally modelling the biophysics of fluorescence spectral emission and microscope performance (Fig 2 A, B, figs. S3-S5). We used this synthetic dataset to evaluate LU and HyU algorithm performance quantitatively by using metrics such as Mean Square Error (MSE) and unmixing residual (see Fig. S6, Methods; for both metrics, a lower value indicates better performance). In addition to the computational efficiency mentioned above, HyU analysis shows better ability to capture spatial features over a wide dynamic range of intensities, when compared with standard LU, in large part due to the denoising created by processing in phasor space (Fig. 2 A, B). The improved accuracy is demonstrated by a lower MSE, in comparing the results of LU and HyU to the image ground truth. The absolute MSE for HyU is consistently up to $2 x$ lower than that of LU, especially at 
low and ultra-low fluorescence levels (Fig. 2C). MSE can be further decreased by the use of denoising filters on the phasor plot, resulting in superiority of HyU relative to LU for HFI at low (5-20 photons/spectrum) and ultralow (2-5 photons/spectrum) levels (Fig. 2D). To better characterize the performance in the experimental data without ground truth, we also define the unmixing residual as the difference between the original multichannel hyperspectral images and their unmixed results. Residuals provide a measure of how closely the unmixed results reconstruct the original signal (Fig. S3, Methods). Unmixing residuals are inversely proportional to the performance of the algorithm, with low residuals indicating high similarity between the unmixed and the original signals. Analysis of unmixing residuals in the synthetic data highlights an improved interpretation of the spectral information in $\mathrm{HyU}$ with an average unmixing residual reduction of 21\% compared to the standard (Fig. S5C). The reduction in both MSE and average unmixing residual for synthetic data demonstrates the superior performance of $\mathrm{HyU}$, and provides a baseline comparison when demonstrating performance improvements for experimental data.

We support the enhanced performance of $\mathrm{HyU}$ with analysis of experimental data, which reveals comparatively lower unmixing residuals and a higher dynamic range as compared to LU. Data was acquired from a quadra-transgenic zebrafish embryo $T g$ (ubiq:Lifeact-mRuby);Gt(cltcacitrine); $\operatorname{Tg}$ (ubiq:lyn-tdTomato);Tg(fli1:mKO2), labelling actin, clathrin, plasma membrane, and pan-endothelial cells, respectively (Figs. 2E-L, 3, S7-S9, Supplementary Movie 1). HyU unmixing of the data shows minimal signal cross-talk between channels while LU presents noticeable bleed-through (Fig. 2M-P). Consistently with synthetic data, we utilize the unmixing residual as the main indicator for quality of the analysis in experimental data, owing to the absence of a ground truth. The residual images (Fig. 2F, G) depict a striking difference in performance between $\mathrm{HyU}$ and LU. The average relative residual of HyU denotes a 7-fold improvement compared to LU (Fig. 2H) in disentangling the fluorescent spectra. We visualize the unmixed channels independently (Fig. 2, I to L), zooming in on details (Fig.2 I to P) to highlight areas affected by bleed-through and which are difficult to unmix. HyU, with contrast 2 -fold higher than standard LU, reduces bleed-through effects and produces images with sharper spatial features, leading to better interpretation of the experimental data (Fig. 2 K, L, fig. S7, Methods). 

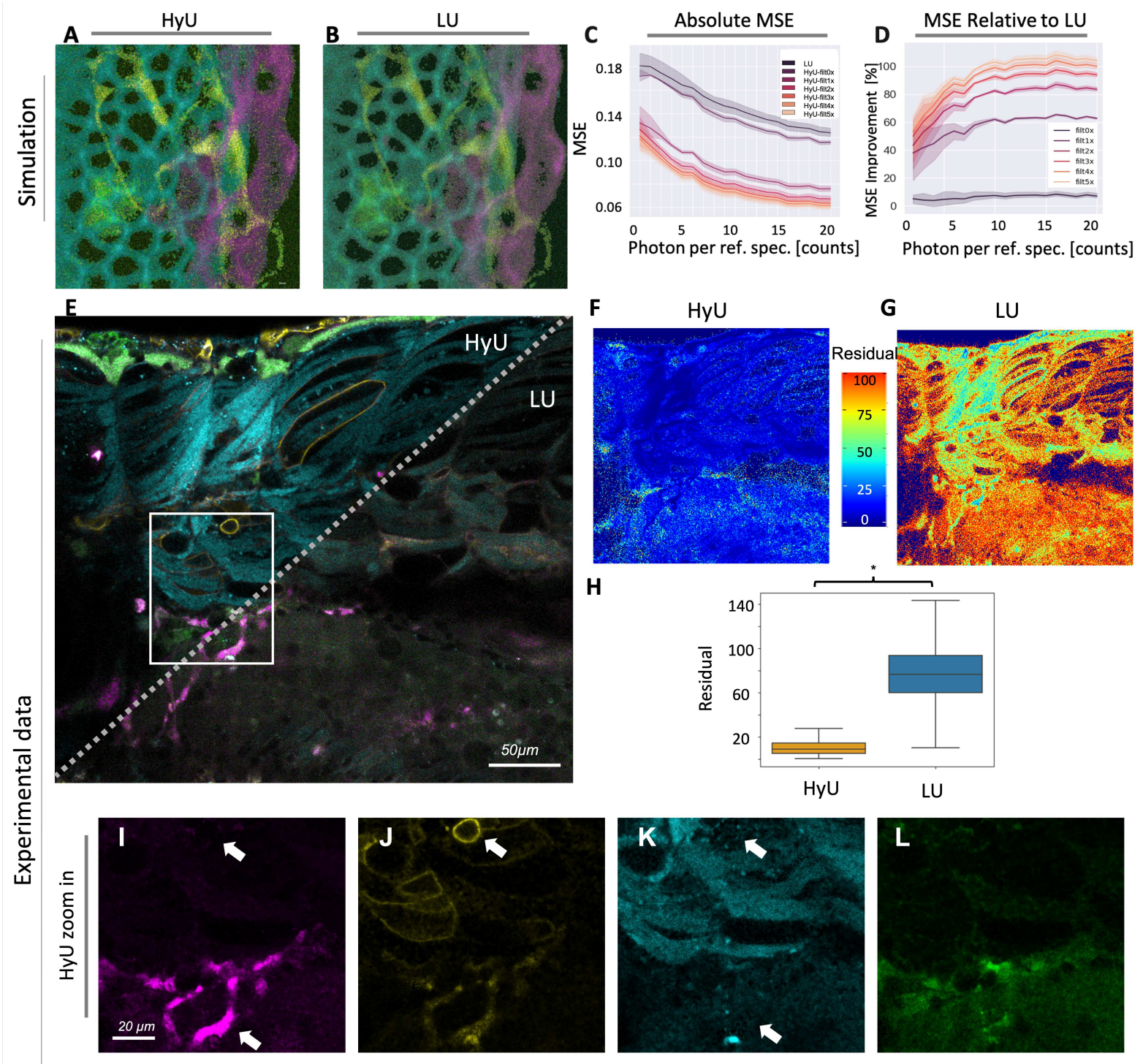

G

LU

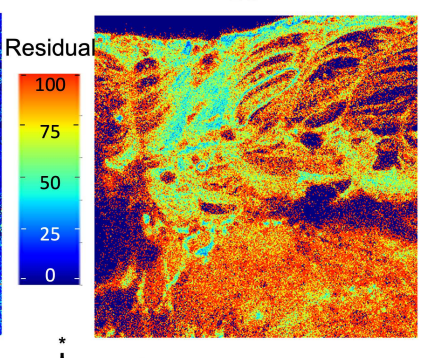

H
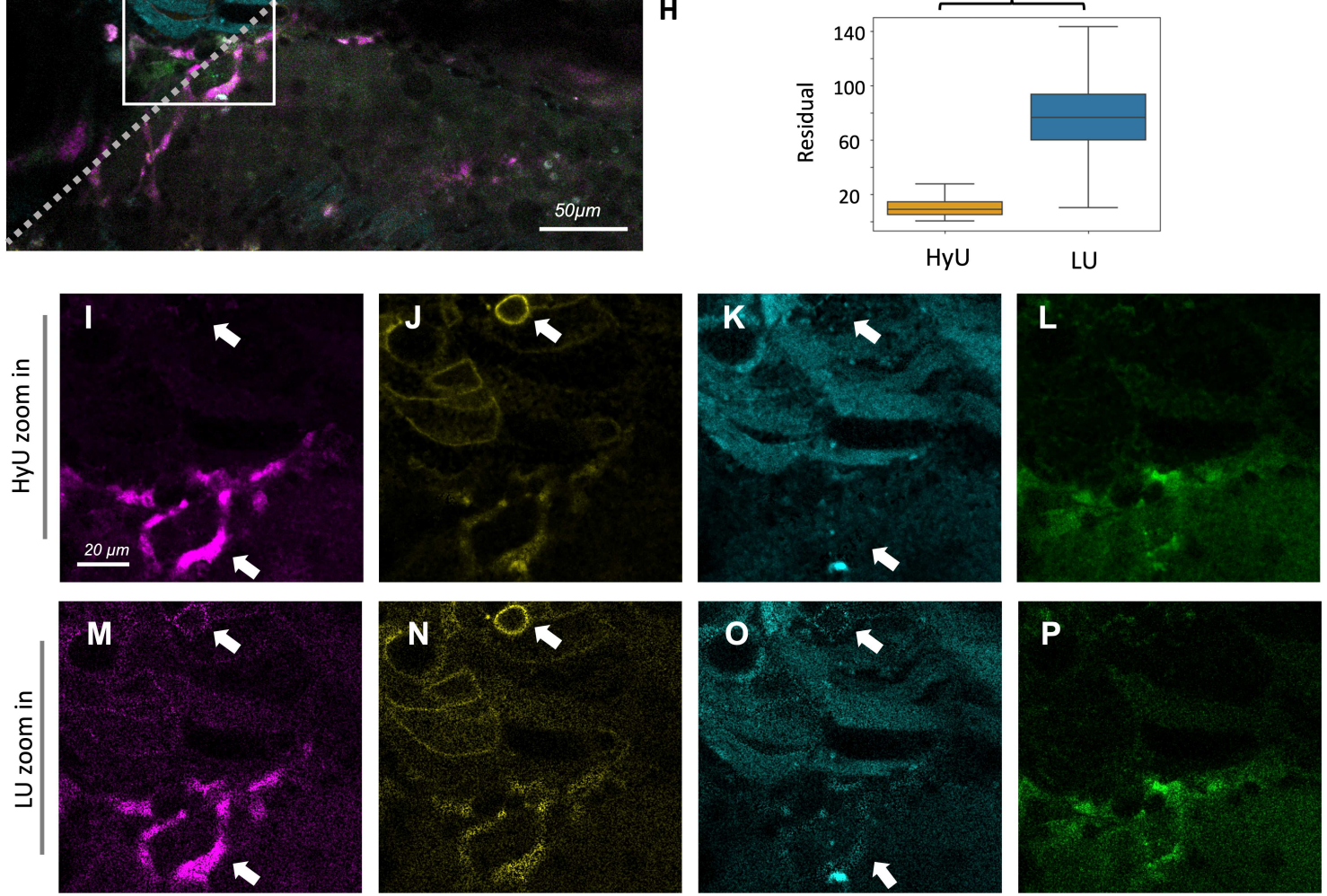

fli1:mKO2

ubiq:lyn-tdTomato

ubiq:Lifeact-mRuby

cltca-Citrine

Figure 2: Hybrid Unmixing outperforms standard Linear Unmixing (LU) in both synthetic and live spectral fluorescence imaging. (A) Hybrid Unmixing (HyU) and (B) Linear Unmixing (LU) tested using a hyperspectral fluorescence simulation that was generated from four fluorescent signatures (emission spectra, Sup fig 5E). (C) Absolute Mean Squared Error (MSE) shows that HyU offers a consistent reduction in error across a broad range of photons per spectra (\#photons/independent spectral components, here resulting from 4 reference spectra combined). (D) The performance differences in the MSE of HyU relative to LU persists when applying multiple phasor denoising filters ( 0 to 5 median filters). The analysis of this synthetic data shows the consistent improvement of $\mathrm{HyU}$ at low photon counts with over a 2-fold improvement when 5 denoising filters are applied at a signal level of 16 photons per spectrum. (E) Unmixing of experimental data from a 4-color zebrafish shows increased contrast for 
HyU (left) compared to LU (right). Scale bar $=50 \mu \mathrm{m}$. (F, G) The increased accuracy is revealed by residual images of $\mathrm{HyU}$ and LU, showing the spatial distribution of unassigned signals after the analysis of data in $\mathrm{E}$. The results show consistently lower residual values for $\mathrm{HyU}(\mathrm{F})$ compared to $\mathrm{LU}(\mathrm{G})$. (H) Box plots of the residuals in $\mathrm{F}$ and $\mathrm{G}$ presents values of $11 \%$ for HyU compared to $77 \%$ for $\mathrm{LU}$ with * $\left(\mathrm{p}<10^{-10}\right)$. (I-L) Enlarged rendering of HyU results (E, white box) clearly shows low levels of bleed-through between labels (M-P) Similar enlargement of LU results show noticeably worse performance. Note that regions with bright signals (membrane $\mathrm{J}, \mathrm{N}$ white arrow) bleed through other channels (M) and (O). Scale bar: $20 \mu \mathrm{m}$. Tetra-labeled specimen used here was Gt(cltcacitrine);Tg(ubiq:lyn-tdTomato; ubiq:Lifeact-mRuby;fli1:mKO2) 

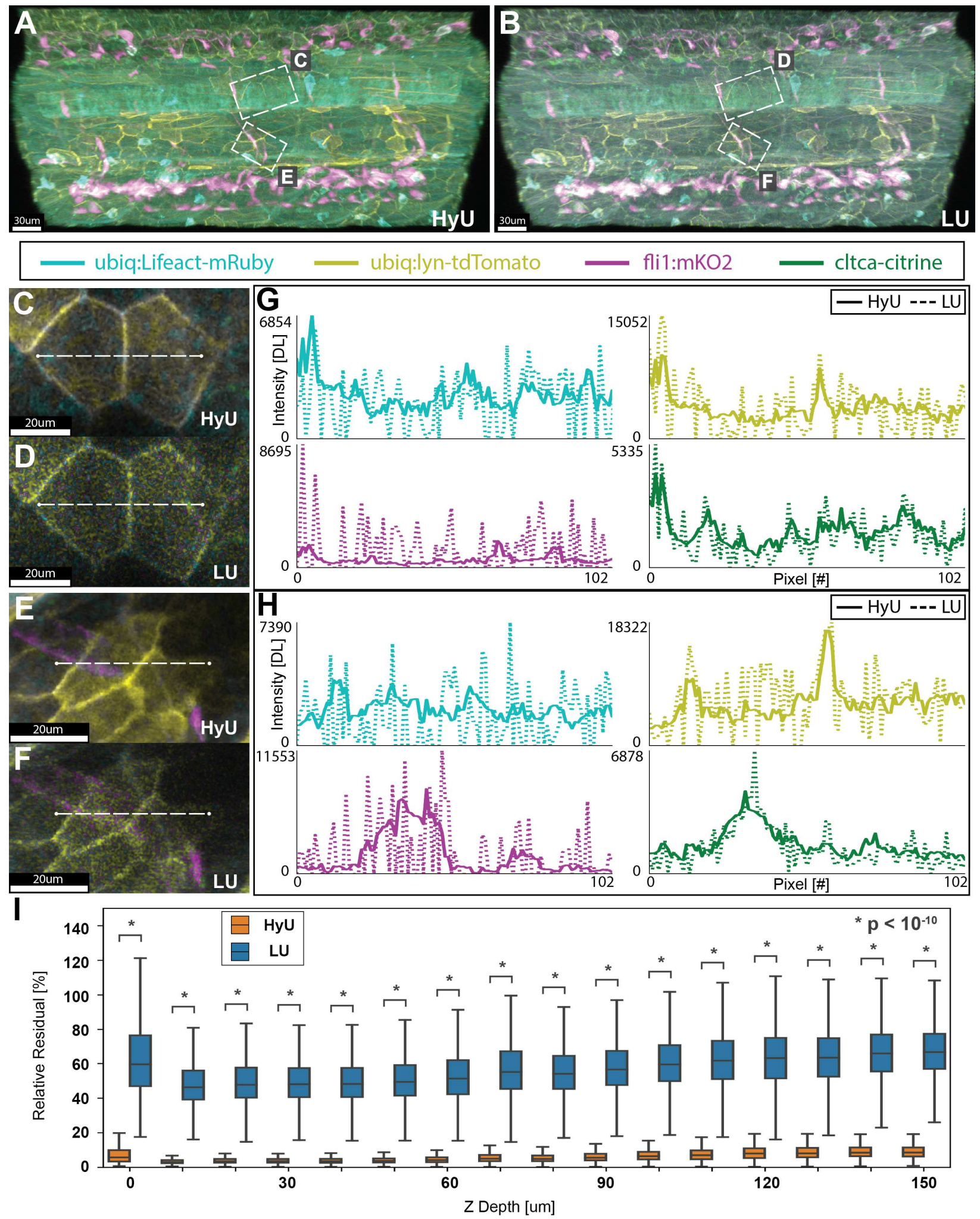

Figure 3: Hybrid Unmixing enhances unmixing for low-signal in vivo multiplexing and achieves deeper volumetric imaging. (A) Hybrid Unmixing (HyU) volumetric renderings compared to those of (B) Linear Unmixing (LU) for the trunk portion in a 4-color zebrafish demonstrate an increased contrast and reduced residual in HyU results, especially over deeper parts of the sample. The 4 labels in the fish are Gt(cltca-citrine); $T g(u b i q: l y n$ tdTomato;ubiq:Lifeact-mRuby;fli1:mKO2), respectively labeling clathrin-coated pits (green), membrane (yellow), 
actin (cyan) and endothelial (magenta). (C,E) HyU results have increased spatial resolution and less bleed though comparing to those of (D,F) LU. Scale bar: $20 \mu \mathrm{m}$. When observing the zoomed-in visualization of the surface region of the sample, the yellow signal distinctly marks the membrane and the cyan signal clearly labels the actin in (C) HyU. The same signals are not distinct in (D) LU because of multiple incorrectly assigned magenta pixels that bleed through compromising the true signal in other channels. Similarly, for the zoomed-in visualization of the Perivascular region of the embryo, in (E) HyU, the yellow and magenta signals clearly distinguish the membrane and vasculature while in (F) LU, the results are corrupted by greater noise. (G,H) Intensity line plots of each of the four results signals for HyU (solid) and LU (dashed) demonstrate the improved profiles with greatly reduced noise peaks in HyU as compared to LU. Intensities are scaled by the maximum of each unmixed channel. DL: digital level. (I) Box plots of the relative residual values as a function of $\mathrm{z}$ depth for $\mathrm{HyU}$ and LU highlight the improvements in the unmixing results. HyU has an unmixing residual of $6.6 \% \pm 5.3 \%$ compared to LU's $58 \% \pm$ $17 \%$. The average amount of residual is 9 -fold lower in $\mathrm{HyU}$ with narrower variance of residual.

Applying HyU to another HFI dataset further highlights HyU's improvements in noise reduction and reconstitution of spatial features for low-photon unmixing. (Figs. 3, S8). In the zoomed-in image of a single slice of the embryo skin surface, acquired in the trunk region, the HyU image correctly does not display pan-endothelial (magenta) signal in the periderm, an area which should be devoid of endothelial cells and mKO2 signal (Fig. 3C). In contrast, the result from LU shows visually distinctive pan-endothelial signal throughout the tissue plane (Fig. 3D). This incorrect estimation of the relative contribution of $\mathrm{mKO} 2$ fluorescence for LU is possibly due to the presence of noise, corrupting the spectral profiles. This is further delineated in the intensity profiles of the $\mathrm{mKO} 2$ signal between $\mathrm{HyU}$ and $\mathrm{LU}$ with much higher individual peaks from noise demonstrated for LU (Fig. 3G, lower left). Intensity profiles for both magnified cross-sections of the volume (Fig. 3C-F) provide a striking visualization of the improvements of HyU. The line intensity profiles in $\mathrm{HyU}$ present reduced noise and represent more closely the expected distribution of signals (Fig. 3G,H). The visible micro patterns of actin on the membrane of the periderm suggest that the improvements quantified with synthetic data are maintained in live samples' signals and geometrical patterns of microridges ${ }^{22}$. By contrast, noise corruption and the presence of misplaced signals are characterized in the results from LU, with high frequency intensity variations that mis-match both the labeling and biological patterns.

$\mathrm{HyU}$ is more accurate and results in more reliable unmixing results across the depth of sample with greatly reduced unmixing residuals. The average residual for $\mathrm{HyU}$ is 9-fold lower than that of LU with a 3-fold narrower variance. (Figs. 3I, S8). This reduction in the residual is consistent with increasing z-depth where HyU unmixing results stably maintain both lower residuals and variance on average. These reduced residuals correspond both to a mathematically more precise and more uniform decomposition of signals as illustrated by the distribution of residuals versus photons (Figs. S8E, F, S14). 

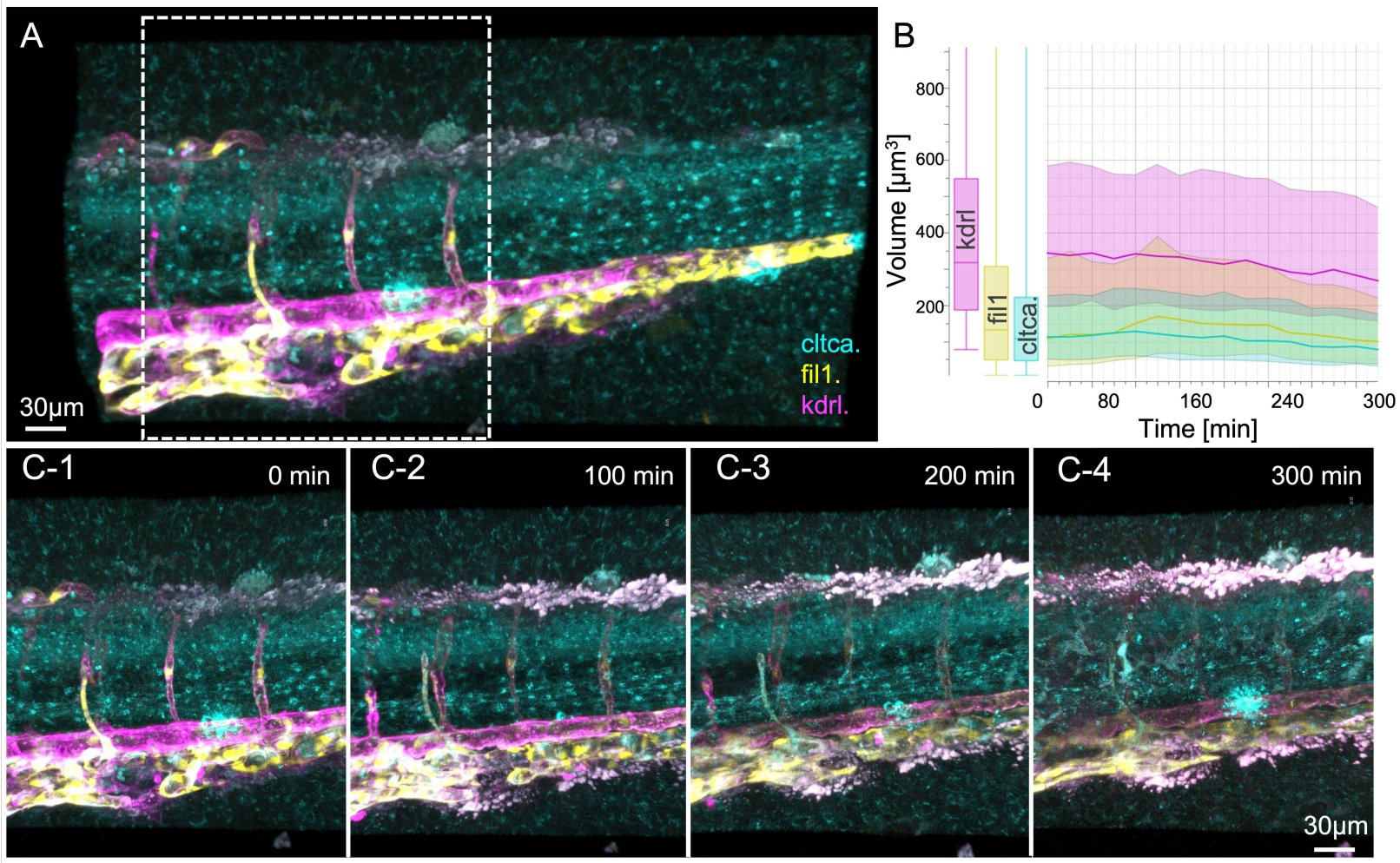

Figure 4: HyU reveals the dynamics of developing vasculature by enabling multiplexed volumetric time-lapse. Hybrid Unmixing (HyU) overcomes challenges in performing multiplexed volumetric time-lapse in vivo imaging of a developing embryo. Here we present this (A) HyU rendering for the trunk portion of a 3-color zebrafish Gt(cltcacitrine); $T g(k d r l: m C h e r r y ; f l i 1: m K O 2)$ at timepoint 0 . (B) HyU unmixed results allow for quantitative analysis and segmentation, here an example representing the time evolution of the segmented volumes of mCherry (vasculature, magenta) mKO2(endothelial-lymphatics, yellow) and citrine (clathrin-coated pits, cyan). (C1-4) Time lapse imaging of the formation of the vasculature over 300 mins (zoomed-in rendering of the box in A) at 0, 100, 200, 300 minutes. This show that HyU provides good unmixing at low light levels to permit multiplexing to be used in the observation of development of a live embryo.

We utilized HyU's increased sensitivity to overcome common challenges of multiplexed imaging such as poor photon yield and spectral cross-talk and were able to visualize dynamics in a developing zebrafish embryo. We used a triple-transgenic zebrafish embryo with labeled panendothelial cells, vasculature, and clathrin-coated pits (Tg(fli1:mKO2); $T g(k d r l: m C h e r r y)$; Gt(cltca-Citrine)). Multiplexing these spectrally close fluorescent proteins is enabled by HyU's increased sensitivity at lower photon counts. The increased performance at lower SNR allowed us to maintain high quality results (Fig. 4, Supplementary Movie 2) while performing faster acquisitions and reducing photon-damage through lower excitation laser power and pixel dwell time. Decreased experimental requirements allow for tiling of larger volumes, extending the field-of-view while still providing enough time resolution for developmental events, even with a high number of multiplexed fluorescent signals. The time-lapses visualize the formation of ventral vasculo-endothelial protrusions acquired in parallel to the development of clathrin and kdrl. HyU enables comparative quantifications of spatio-temporal features, allowing for the 
determination of volumetric changes over lengthy timelapses, in this case, over the course of 300 minutes (Fig. 4B) ${ }^{23,24}$.

HyU provides the ability to combine the information from intrinsic and extrinsic signals during live imaging of samples, at both single (Fig. 5) and multiple time points (Fig. 6). The graphical representation of phasors allows identification of unexpected intrinsic fluorescence signatures in a quadra-transgenic zebrafish embryo Gt(cltca-citrine); Tg(ubiq:lyn-tdTomato;ubiq:LifeactmRuby;flil:mKO2), imaged with single photon (488 and 561nm excitation) (Fig. 5A-D). The elongated distribution on the phasor (Fig. 5C) highlights the presence of an additional, unexpected spectral signature, related to strong sample autofluorescence (Fig. 5D blue). HyU analysis of the sample, inclusive of this additional signal, provides separation of the contributions of 5 different fluorescent spectra with residual $3.9 \% \pm 0.3 \%$. HyU allows for reduced energy load, tiled imaging of the entire embryo without perturbing its development or depleting its fluorescence signal (Fig. 5A). The higher speed, lower power imaging allows for subsequent re-imaging of the same sample, as we report in the zoomed high-resolution acquisitions of the head section (Fig. 5B,E).

With the ability to unmix low photon signals, HyU enables imaging and decoding of intrinsic signals, which are inherently low light. Two photon lasers are ideal for exciting and imaging blue-shifted intrinsic fluorescence from samples ${ }^{25,26}$. Here, the same quadra-transgenic sample is imaged using $740 \mathrm{~nm}$ excitation to access both intrinsic and extrinsic signals (Fig $5 \mathrm{E}-\mathrm{G}$, sup Note 2). HyU enables unmixing of at least 9 intrinsic and transgenic fluorescent signals (Fig. 5), recovering fluorescent intensities from labels illuminated at a sub-optimal excitation wavelength (Fig. 5E). The spectra for intrinsic fluorescence were obtained from in vitro measurements and values reported in literature (Methods). For this sample the intrinsic signals arise from events related mainly with metabolic activity (NADH and Retinoids) $)^{27-31}$, tissue structure (elastin) ${ }^{32}$, and illumination (laser reflection) (Fig. 5E). These results confirm our conclusion that $\mathrm{HyU}$ is a powerful tool for allowing the imaging and analysis of endogenous labels. 


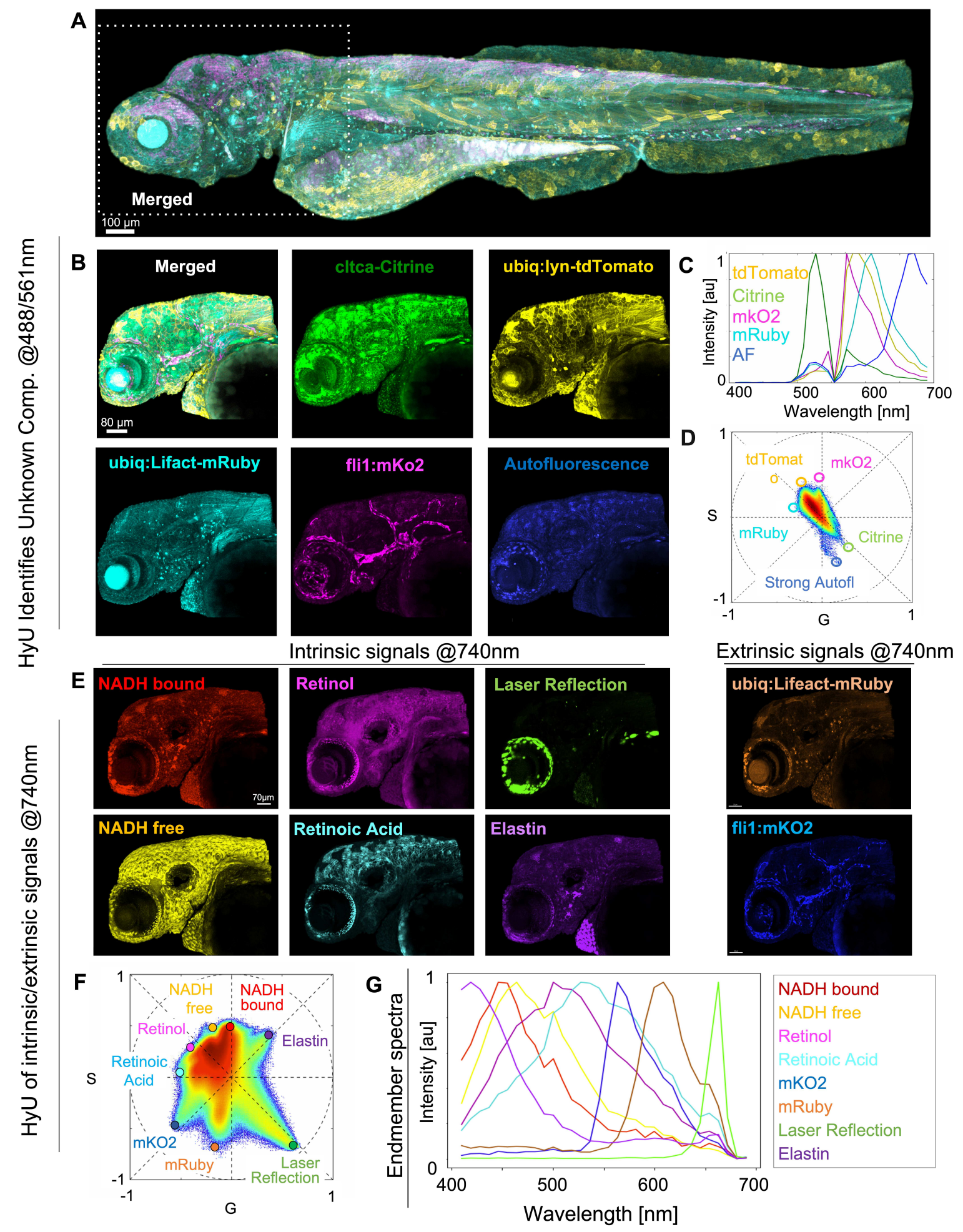

Figure 5: HyU enables identification and unmixing of low photon intrinsic signals in conjunction with extrinsic signals. (A) HyU results of a whole zebrafish embryo provide a frame of reference not only for the improved unmixing of extrinsic signals, but also its increased sensitivity which enables identification and unmixing of intrinsic signals which inherently exist in a low-photon environment. (B) HyU results of the head region (box in A) reveal the simplicity of identifying an unknown autofluorescent signal among multiple extrinsic signals using the phasor method for a quadra-transgenic zebrafish Gt(cltca-citrine); $\operatorname{Tg}$ (ubiq:lyn-tdTomato;ubiq:Lifeact- 
mRuby;fli1:mKO2) imaged over multiple tiles. Scale bar: $80 \mu \mathrm{m}$. (C) The input spectra required to perform the unmixing are easily identified on (D) the phasor plot when visualizing each spectrum as a spatial location. Phasors offer a simplified identification and selection of independent and unexpected spectral components in the encoded HyU approach. Intrinsic signals are notoriously low in emitted photons leading to an inability to unmix using traditional unmixing algorithms. (E) The zoomed-in acquisition of the head region of the embryo (box in A) displays HyU's unmixing results of many intrinsic and extrinsic signals when in an environment of very low photon output, a previously highly difficult experimental condition to unmix. Scale bar: $70 \mu \mathrm{m}$. (F) The phasor plot representation provides the easily identifiable eight independent fluorescent fingerprint locations. (G) The spectra corresponding to each of the eight independent spectral components are also provided a reference. Colors in (F) match renderings in (E) and (G): NADH bound (red), NADH free (yellow), retinoid (magenta), retinoic acid (cyan), reflection (green), elastin (purple) and extrinsic signals: $\mathrm{mKO} 2$ (blue), and mRuby (orange). All signals were excited with a (A-D) single photon laser at both $488 \mathrm{~nm}$ and $561 \mathrm{~nm}$ or a (E-G) two photon laser at $740 \mathrm{~nm}$. 
Intrinsic signals @740nm Ex.

Extrinsic signals @488/561nm Ex.

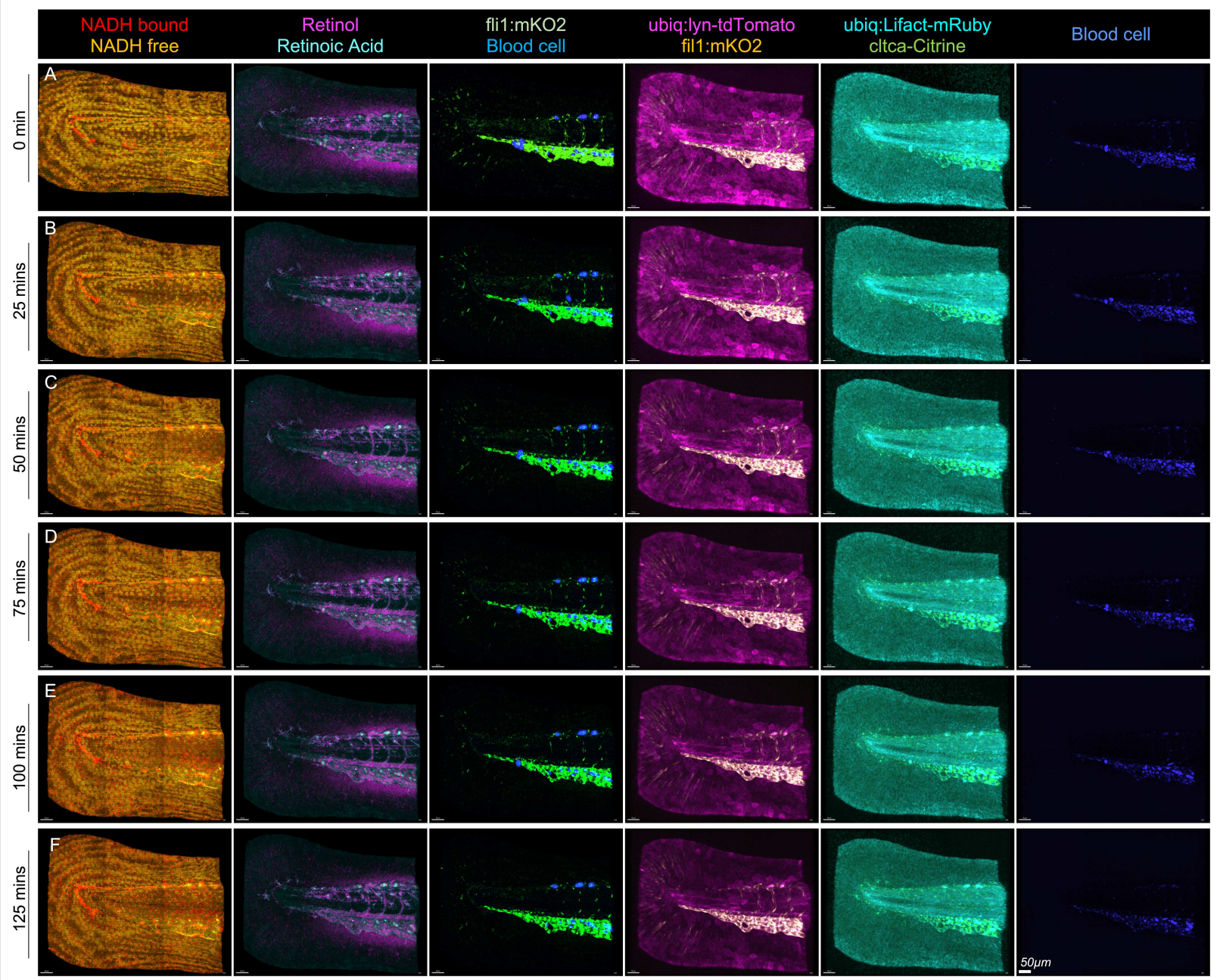

Figure 6: HyU pushes the upper limits of live multiplexed volumetric timelapse imaging of intrinsic and extrinsic signals.

HyU's increased sensitivity provides a simple solution for the challenging task of imaging timelapse data at 6 time points (125 mins) for both intrinsic signals and extrinsic signals of a quadra-transgenic zebrafish: $\operatorname{Tg}((\mathrm{cltca}-$ Citrine);(ubiq:lyn-tdTomato);(ubiq:Lifeact-mRuby);(fli1:mKO2)). (A) - (F) Volumetric renderings of HyU results for time points acquired at $25 \mathrm{~min}$ intervals reveal the high-contrast and -multiplexed labels of NADH bound (red), NADH free (yellow), retinoid (magenta), retinoic acid (cyan), mKO2 (green), and autofluorescence from blood cells (blue) when excited@740nm. Further extrinsic signals for mKO2 (yellow), tdTomato (magenta), mRuby (cyan), Citrine (green) and blood cells autofluorescence (blue) are also readily unmixed using HyU when exciting the sample@488/561nm. HyU provides the capacity to simultaneously multiplex 9 signals in a live sample over long periods of time, a previously unexplored task. Scale bar: $50 \mu \mathrm{m}$.

Finally, we exploited the HyU capabilities to multiplex volumetric timelapse of extrinsic and intrinsic signals by imaging the tail region of the same quadra-transgenic zebrafish embryo. We excite extrinsic labels at $488 / 561 \mathrm{~nm}$ and the intrinsic signals with $740 \mathrm{~nm}$ two photon, collecting 
6 tiled volumes over 125 mins (Figs. 6, S9-S11, Supplementary Movie 3). HyU unmixing in this sample allows for distinction of 9 signals, separating their contributions with sufficiently low requirements to allow repeated imaging of notoriously low SNR intrinsic fluorescence.

\section{Discussion}

Our results reveal the advantages of Hybrid Unmixing (HyU) over more conventional Linear Unmixing (LU) in performing complex multiplexing experiments. HyU overcomes the significant challenges of separating multiple fluorescent and autofluorescent labels with overlapping spectra while minimally perturbing the sample with excitation light.

The chief advantage of $\mathrm{HyU}$ is its multiplexing capability when imaging in the presence of biological and instrumental noise, especially at low signal levels. HyU increased sensitivity improves multiplexing in photon limited applications (Fig. 2F-L), in deeper volumetric acquisitions (Fig. 3I) and in signal starved imaging of autofluorescence (Fig. 5E, Fig. 6). Our simulation results (Fig. 2) demonstrate that HyU improves unmixing of spatially and spectrally overlapping fluorophores excited simultaneously. The increased robustness at low photon imaging conditions reduces the imaging requirements for excitation levels and detector integration time, allowing for imaging with reduced photo-toxicity. Live imaging on multi-color samples performed at high sampling frequency enables improved tiling to increase the field-ofview (Fig. 3, 4) while maximizing the usage of the finite fluorescent signals over time. Twophoton imaging of intrinsic and extrinsic signals suggests the ability of $\mathrm{HyU}$ to multiplex signals with large dynamic range differences (Fig. 5) extending multiplexed volumetric imaging into the time dimension (Fig. 6). Although improved, images with particularly low signal still present corruption (Fig. S4), setting a reasonable range of utilization above 8 photons/spectrum.

Simplicity of use and versatility are other key advantages of $\mathrm{HyU}$, inherited from both the phasor approach $^{33}$ and traditional unmixing algorithms. Phasors here operate as a spectral encoder, reducing computational load and integrating similar spectral signatures in histogram bins of the phasor plot. This representation simplifies identification of independent spectral signatures (Fig. 5, Supplementary Note1) through both phasor plot selection and phasor residual mapping (fig. S11), accounting for unexpected intrinsic signals (Figs. 5, 6, S12, Supplementary Note2) in a semi-automated manner, while still allowing fully-automated analysis by means of spectral libraries.

The simplicity of this approach is especially helpful in live imaging where identifying independent spectral components remains an open challenge, owing to the presence of intrinsic signals (Fig. S12, Supplementary Note 1). High-SNR reference spectra can be derived from other experimental data or identified directly on the phasor. Selection of portions on the phasor plot allows for visualization of the corresponding spectra in the wavelength domain (Fig $5 \mathrm{C}, \mathrm{D}, \mathrm{F}, \mathrm{G})$. This intuitive versatility allows for identification of both the number of unexpected signatures and their spectra, a task previously difficult to perform due to noise and lack of global visualization tools. In single photon imaging (Fig. 5A-D), HyU phasor allowed identification of a fifth distinct spectral component arising from general autofluorescent background, thereby improving the unmixed results. In two photon imaging, HyU enabled identification and multiplexing of 8 highly overlapping signals possessing a wide dynamic range of intensities, between intrinsic and extrinsic markers (Fig. 5F,G). Combination of single and two photon imaging increased the number of multiplexed fluorophores to 9 (Fig. 6), considering some of the 
extrinsic labels being excited at two photons. Multiplexing of signals may be further improved by implementing HyU on fluorescent dyes.

HyU performs better than standard algorithms both in the presence and absence of phasor noise reduction filters ${ }^{33}$. Compared with LU, the unmixing enhancement when such filters ${ }^{33}$ are applied is demonstrated by a decrease of the MSE of up to $21 \%$ (Fig 2C), with a reduction of the average amount of residuals by 7 -fold. Even in the absence of phasor denoising filters, HyU performs up to $7.3 \%$ better than the standard (Fig. 2D) based on Mean Squared Error of synthetic data unmixing. This base improvement is due to the averaging of similarly shaped spectra in each phasor histogram bin, which reduces the statistical variability within the spectra used for the unmixing calculations (Fig. 1E). This averaging strategy works well for general fluorescence spectra owing to their broad and mostly unique spectral shape.

In the absence of noise, for example in the ground truth simulations, LU produces an MSE 6-fold lower than HyU (Fig. S5, B, C, S6G). In these noiseless conditions, the binning and averaging of spectra in the phasor histogram, without denoising, provides statistically indifferent values of error respect to LU, suggesting results of similar quality.

HyU can interface with different unmixing algorithms, adapting to existing experimental pipelines. We successfully tested hybridization with iterative approaches such as non-negative matrix factorization ${ }^{34}$, fully constrained and non-negative least-squares ${ }^{35}$ (Methods). Speed tests with iterative fitting unmixing algorithms demonstrate a speed increase of up to 500-fold when the HyU compressive strategy is applied. (Fig. S13, Supplementary Note 3). Due to the initial computational overhead for encoding spectra in phasors, there is a 2-fold speed reduction for HyU in comparison to standard LU. However, this may be improved with further optimizations of the HyU implementation.

One restriction of $\mathrm{HyU}$ derives from the mathematics of linear unmixing, where linear equations representing the unmixed channels need to be solved for the unknown contributions of each analyzed fluorophore. To obtain a unique solution from these equations and to avoid an underdetermined equation system, the maximum number of spectra for unmixing may not exceed the number of channels acquired ${ }^{36}$, generally 32 for commercial microscopes. This number could be increased; however, due to the broad and photon-starved nature of fluorescence spectra, acquisition of a larger number of channels could negatively affect the sample, imaging time and intensities. Depending on the number of labels in the specimen of interest, extending the number of labels to simultaneously unmix beyond 32 will likely require spectral resolution upsampling strategies.

$\mathrm{HyU}$ improvement is related to the presence of various types of noise in microscopy images, such as Gaussian, Poisson and digital as well as unidentified sources of spectral signatures (Fig. $\mathrm{S} 5 \mathrm{~B}, \mathrm{C}, \mathrm{S} 6 \mathrm{G})$. In the multiplexing of fluorescent signals, HyU offers improved performance, quality- and speed-wise in the low-signal regime. HyU is poised to be used in the context of in vivo imaging, harvesting information from samples labeled at endogenous-level.

In conclusion, the results presented in this paper quantitatively show that HyU, a phasor based, computational unmixing framework, is well suited for tackling the many challenges present in live imaging of multiple fluorescence labels. HyU's reduced requirements in amount of 
fluorescent signal permit a reduction of laser excitation load and imaging time. These factors enable multiplexed imaging of biological events with longer duration, higher speed and lower photo-toxicity while providing access to information-rich imaging across different spatiotemporal scales. The reduced requirements of HyU make it fully compatible with any commercial and common microscopes capable of spectral detection, facilitating access to the technology. Our analysis demonstrates HyU's robustness, simplicity and improvement in identifying both new and known spectral signatures, and vastly improved unmixing outputs, providing a much-needed tool for delving into the many questions still surrounding studies with live imaging. 


\section{References and Notes:}

1. Valm, A. M. et al. Applying systems-level spectral imaging and analysis to reveal the organelle interactome. Nature 546, 162-167 (2017).

2. Tsurui, H. et al. Seven-color fluorescence imaging of tissue samples based on fourier spectroscopy and singular value decomposition. Journal of Histochemistry and Cytochemistry 48, 653-662 (2000).

3. Amat, F. et al. Fast, accurate reconstruction of cell lineages from large-scale fluorescence microscopy data. Nature Methods 11, 951-958 (2014).

4. Ueno, T. \& Nagano, T. Fluorescent probes for sensing and imaging. Nature Methods vol. 8 642-645 (2011).

5. Lichtman, J. W. \& Conchello, J. A. Fluorescence microscopy. Nature Methods vol. 2 910-919 (2005).

6. Sinclair, M. B., Haaland, D. M., Timlin, J. A. \& Jones, H. D. T. Hyperspectral confocal microscope. 45, 6283-6291 (2006).

7. Jahr, W., Schmid, B., Schmied, C., Fahrbach, F. O. \& Huisken, J. Hyperspectral light sheet microscopy. Nature Communications 6, 1-7 (2015).

8. Truong, T. V, Supatto, W., Koos, D. S., Choi, J. M. \& Fraser, S. E. Deep and fast live imaging with two-photon scanned light-sheet microscopy. 8, (2011).

9. Chen, B. C. et al. Lattice light-sheet microscopy: Imaging molecules to embryos at high spatiotemporal resolution. Science 346, (2014).

10. Kredel, S. et al. mRuby, a bright monomeric red fluorescent protein for labeling of subcellular structures. PLOS ONE 4, 1-7 (2009).

11. Sakaue-Sawano, A. et al. Visualizing Spatiotemporal Dynamics of Multicellular CellCycle Progression. Cell 132, 487-498 (2008).

12. Wade, O. K. et al. 124-Color Super-resolution Imaging by Engineering DNA-PAINT Blinking Kinetics. Nano Letters 19, 2641-2646 (2019).

13. Strauss, S. \& Jungmann, R. Up to 100 -fold speed-up and multiplexing in optimized DNAPAINT. Nature Methods 17, 789-791 (2020).

14. Zimmermann, T., Rietdorf, J. \& Pepperkok, R. Spectral imaging and its applications in live cell microscopy. FEBS Letters 546, 87-92 (2003).

15. Paddock, S. Multi-Spectral Imaging and Linear Unmixing Add a Whole New Dimension to. BioTechniques 31, 1272-1278 (2001).

16. Zimmermann, T. Spectral imaging and linear unmixing in light microscopy. Advances in Biochemical Engineering/Biotechnology 95, 245-265 (2005).

17. Garini, Y., Young, I. T. \& McNamara, G. Spectral imaging: Principles and applications. Cytometry Part A vol. 69 (2006).

18. Digman, M. A., Caiolfa, V. R., Zamai, M. \& Gratton, E. The Phasor Approach to Fluorescence Lifetime Imaging Analysis. Biophysical Journal 94, L14-L16 (2008).

19. Fereidouni, F., Bader, A. N., Colonna, A. \& Gerritsen, H. C. Phasor analysis of multiphoton spectral images distinguishes autofluorescence components of in vivo human skin. Journal of Biophotonics 7, 589-596 (2014).

20. Scipioni, L., Rossetta, A., Tedeschi, G. \& Gratton, E. Phasor S-FLIM: a new paradigm for fast and robust spectral fluorescence lifetime imaging. Nature Methods 18, 542-550 (2021).

21. Ranjit, S., Malacrida, L., Jameson, D. M. \& Gratton, E. Fit-free analysis of fluorescence lifetime imaging data using the phasor approach. Nature Protocols 13, 1979-2004 (2018). 
22. Depasquale, J. A. Actin Microridges. Anatomical record (Hoboken, N.J. : 2007) 301, 2037-2050 (2018).

23. Okuda, K. S., Hogan, B. M., Cantelmo, A. R. \& Hogan, B. M. Endothelial Cell Dynamics in Vascular Development : Insights From Live-Imaging in Zebrafish. 11, (2020).

24. Isogai, S., Lawson, N. D., Torrealday, S., Horiguchi, M. \& Weinstein, B. M. Angiogenic network formation in the developing vertebrate trunk. (2003) doi:10.1242/dev.00733.

25. Denk, W., Strickler, J. H. \& Webb, W. W. Two-photon laser scanning fluorescence microscopy. Science 248, (1990).

26. Zipfel, W. R. et al. Live tissue intrinsic emission microscopy using multiphoton-excited native fluorescence and second harmonic generation. Proceedings of the National Academy of Sciences 100, 7075-7080 (2003).

27. Bird, D. K. et al. Metabolic mapping of MCF10A human breast cells via multiphoton fluorescence lifetime imaging of the coenzyme NADH. Cancer Research 65, (2005).

28. Lakowicz, J. R., Szmacinski, H., Nowaczyk, K. \& Johnson, M. L. Fluorescence lifetime imaging of free and protein-bound NADH. Proceedings of the National Academy of Sciences of the United States of America 89, (1992).

29. Skala, M. C. et al. In vivo multiphoton microscopy of NADH and FAD redox states, fluorescence lifetimes, and cellular morphology in precancerous epithelia. Proceedings of the National Academy of Sciences of the United States of America 104, 19494-19499 (2007).

30. Sharick, J. T. et al. Protein-bound NAD(P)H Lifetime is Sensitive to Multiple Fates of Glucose Carbon. Scientific Reports 8, (2018).

31. Stringari, C. et al. Phasor approach to fluorescence lifetime microscopy distinguishes different metabolic states of germ cells in a live tissue. Proceedings of the National Academy of Sciences of the United States of America 108, (2011).

32. Wagnieres, G. A., Star, W. M. \& Wilson, B. C. Invited Review ln Vivo Fluorescence Spectroscopy and Imaging for Oncological Applications. 68, 603-632 (1998).

33. Cutrale, F. et al. Hyperspectral phasor analysis enables multi- plexed 5D in vivo imaging. Nature Publishing Group (2017) doi:10.1038/nmeth.4134.

34. Févotte, C. \& Dobigeon, N. Nonlinear hyperspectral unmixing with robust nonnegative matrix factorization. IEEE Transactions on Image Processing 24, (2015).

35. Heslop, D., von Dobeneck, T. \& Höcker, M. Using non-negative matrix factorization in the "unmixing" of diffuse reflectance spectra. Marine Geology 241, 63-78 (2007).

36. Paddock, S. W. Confocal Microscopy, Methods and Protocols, Second Edition. Humana Press 1075, 388 (2014).

Acknowledgments: The authors would like to thank F. Schneider, S. Restrepo (Translational Imaging Center, University of Southern California), Chi-Li Chiu (Bitplane Inc.) and Samuel Ojosnegros (Institute for Bioengineering of Catalonia) for helpful discussions.

Funding: This material is based upon work supported by the National Science Foundation Graduate Research Fellowship under Grant DGE-1842487, Department of Defense PR150666 and University of Southern California. 
Author contributions: H.J.C., D.E.S.K., and F.C. analyzed the results and wrote the software. H.J.C., D.E.S.K., J.U. and F.C. provided conceptualization. H.J.C., D.E.S.K., M.K., F.C, and L.A.T. helped in the experimental design and data analysis. M.K. generated the inducible and mKO2 zebrafish lines. H.J.C. and F.C. acquired data. S.E.F. provided supervision. H.J.C., D.E.S.K., and F.C. wrote the paper. L.A.T., J.U., M.K. supported review and editing.

Competing interests: The University of Southern California has filed a provisional patent application covering this method.

Data and materials availability: All the relevant data are available from the corresponding author upon reasonable request. Datasets for Figs. 1-6 and simulations are available for download at http://bioimaging.usc.edu/software.html\#sampledatasets in the samples section. All the relevant code is available from the corresponding author upon reasonable request. Software and instructions can be downloaded from http://bioimaging.usc.edu/software.html. 


\section{Supplementary Files}

This is a list of supplementary files associated with this preprint. Click to download.

- SupplementaryMovie1v3.mp4

- SupplementaryMovie24.mp4

- SupplementaryMovie3v3.mp4

- SupplementaryMovieCaptions211108DKFC.pdf

- HybUnmSupplementaryMaterialsNM20211111FC.pdf

- README2021201FC.pdf 